\title{
UN CUADRO DE ANDREA LILIO EN ESPAÑA
}

El fabuloso Alcázar de Sevilla, reino de la sublime elegancia abstracta de estucos y azulejos mudéjares, conserva un número exiguo de cuadros, generalmente erráticos y aportados allí por vicisitudes y fuentes bastante diversas, aún hoy día en parte misteriosas.

Por esta razón, uno se queda impresionado, cuando entra en la actual Capilla del Palacio Gótico, por un gran retablo [fig.1], colgado en una pared lateral de la sala, de una única nave, de colores luminosos y de trazo tortuoso, en contraste con las sombras y silencios del edificio medieval.

El cuadro recibe la atribución genérica de "Anónimo italiano del siglo XVII", referencia que aparece también en la ficha del catálogo del Patrimonio Nacional, de quien dependen los cuadros y el mobiliario del Alcázar .

11 BoströM, cit.- LAPI BALLERINI, Isabella, Fiorenza,cat.2, Giambologna, gli dei, gli eroi, cit.

12 Dibujos de Arquitectura y Ornamentación de la Biblioteca Nacional Siglos XVI y XVII Madrid., 1991. Catálogo por Elena Santiago. Texto sobre Álbum de Casale, Fernando Marías y Agustín Bustamante. Cat. c19 (el de Pozzuolo), c21.

${ }^{1}$ La tela mide 298x200 (328x230 con el marco); en el catálogo del Patrimonio Nacional es el n ${ }^{\circ}$ PI-17810, inventario $\mathrm{n}^{\circ}$ 10020816. Debo todas las informaciones técnicas relativas a la obra a la gentil colaboración de Carmen García-Frías 
Sin embargo, la obra es reconocible a primera vista como un trabajo de Andrea Lilio de Ancona, pintor que en los últimos veinte años ha recibido una indudable atención crítica, gracias a sus innegables dotes de colorista y de dibujante ${ }^{2}$.

Como se ha dicho, se trata de una tela de gran dimensión, que representa la Virgen coronada, recibida en el cielo por la Trinidad entre ángeles músicos. Estos, tocan o sostienen instrumentos musicales representados con una gran adhesión a la realidad: dos, en un primer plano, un laúd y un arpa, la pareja a los lados de la Virgen, órganos, mientras que en el fondo uno toca el trombón y otros dos leen partituras, también estos retratados con realismo; en la parte más elevada del cuadro dos ángeles con las alas desplegadas hacen oscilar incensarios.

Las comparaciones con la obra de Lilio son muy elocuentes y en mi opinión del todo convincentes, sobre todo con obras realizadas en Las Marcas en los últimos años del siglo: los fragmentos del desmembrado retablo de San Nicola da Tolentino, firmado y fechado en 1598 (hoy en la Pinacoteca Comunale de Ancona), en particular los ángeles músicos [fig.2], los Cuatro santos con ángeles músicos [fig.3], proveniente de la iglesia de I Minori di San Francesco en Alto di Ancona (también en la Pinacoteca Comunale), fechado por Arcangeli y por la crítica hacia los últimos cinco años del siglo XVI, y la Asunción de la Virgen entre santos [fig.4] de la capilla Nolfi del Duomo de Fano, de dudosa datación, pero probablemente ésta también de hacia finales del siglo ${ }^{3}$. La elección cromática, rica en tornasolados y colores frescos y discordantes, permanece dentro del siglo XVI, antes del cambio de Lilio hacia el estilo antitético del tenebrismo caravaggesco y de la nitidez clasicista, ambos utilizados por el artista a partir del primer decenio del XVII.

El problema, frente a una evidente autoría, surge en cambio cuando se trata de identificar la procedencia de la obra, que no pertenece ciertamente a las salas del Alcázar, vista la colocación claramente impropia y las dimensiones de un auténtico retablo.

La documentación moderna conservada en el Patrimonio registra la obra a partir de mayo de 1931, cuando, inmediatamente después de la instauración de la II República española, se redactó un catálogo de pinturas presentes en el Alcázar, antes de que este último pasase de ser propiedad de la Corona española a ser propiedad del Ayuntamiento de Sevilla, es decir, a las autoridades municipales ${ }^{4}$. Pero, ¿y anteriormente?

Guías y descripciones del siglo XIX del Alcázar y en general de la ciudad de Sevilla parecerían ofrecer solo informaciones negativas, partiendo de Noticia artística de Félix González de León, de 1844, donde, en la detallada descripción del edificio y de la capilla no hay rastro de la pintura, hasta Sevilla monumental y artística de Gestoso y Pérez, de los últimos años del XIX, en el que aún no aparece en la capilla real el cuadro de Lilio ${ }^{5}$.

Checa, Fernando A. Martín García, José Luis Sancho Gaspar y Rocío Ferin del Patrimonio Nacional. Además deseo agradecer a Carlos José Hernando Sánchez de la Universidad de Vallodolid y a Francisco Ollero Lobato de la Universidad Pablo de Olavide de Sevilla, por su preciosa ayuda y disponibilidad, y a Gaetano Sabatini, sin cuya ayuda no habría podido escribir esta aportación.

${ }^{2}$ Sobre Andrea Lilio hay mucha bibliografía reciente, me limitaré por tanto a recordar los estudios más importantes, como punto de partida para la elaboración del catálogo pictórico del artista: Andrea Lilli nella pittura delle Marche tra Cinque e Seicento, cat. mostra Ancona, 1985, Roma 1985; ArCAngeli, L., Andrea Lilli, en Dal Poggetto, P., a cargo de Le arti nelle Marche al tempo di Sisto V, cat. exposición, Ascoli Piceno 1992, Milán 1992, pp. 331-335; por último la monografia de Pulini, M., Andrea Lilio, Milán 2003 (con bibl. incluida).

3 Para todas las comparaciones citadas véase Pulini, M., op. cit., pp. 158-165.

${ }^{4}$ Hoy el palacio está constituido en organismo municipal autónomo, con el título de Patronato del Real Alcázar. En el inventario conservado en el Patrimonio Nacional en el Palacio Real de Madrid, redactado el 25 de mayo de 1931, con el n³55 está censado "Otro [lienso] idem con marco liso, de la Asunción con la Trinidad del siglo XVII"; en otro inventario, de poco después (1 de noviembre de 1931), conservado en el Archivo Municipal de Sevilla (Hacienda, 30/31; A/1147), y hecho compilar por el recién elegido conservador Alfonso Lasso de la Vega, con el n ${ }^{\circ} 215$ (p. 10) es nuevamente recordado entre los "otros enseres de la capilla ... un lienzo con marco liso: la Asunción con la Trinidad - siglo XVII", valorado en 1000 pesetas. Agradezco a Inmaculada Franco del Archivo Municipal de Sevilla por la ayuda en la búsqueda.

5 Para las descripciones del siglo XIX del Alcázar, véase GonZÁlez De LEÓN, F., Noticia artística de todos los edificios públicos de esta muy noble ciudad de Sevilla por Don Félix González de León, 2 vol., Sevilla 1844, I, p. 137; Gestoso y PÉrez, J., Sevilla monumental y artística, 3 vol., Sevilla 1889-1897, I, 1889, pp. 293-403. En esta última descripción de la capilla se recuerda ya la copia sobre el altar de la Virgen de la Antigua de la catedral y otros cinco cuadros en la sacristía, entre los que, sin embargo, no figura la tela de Lilio. 


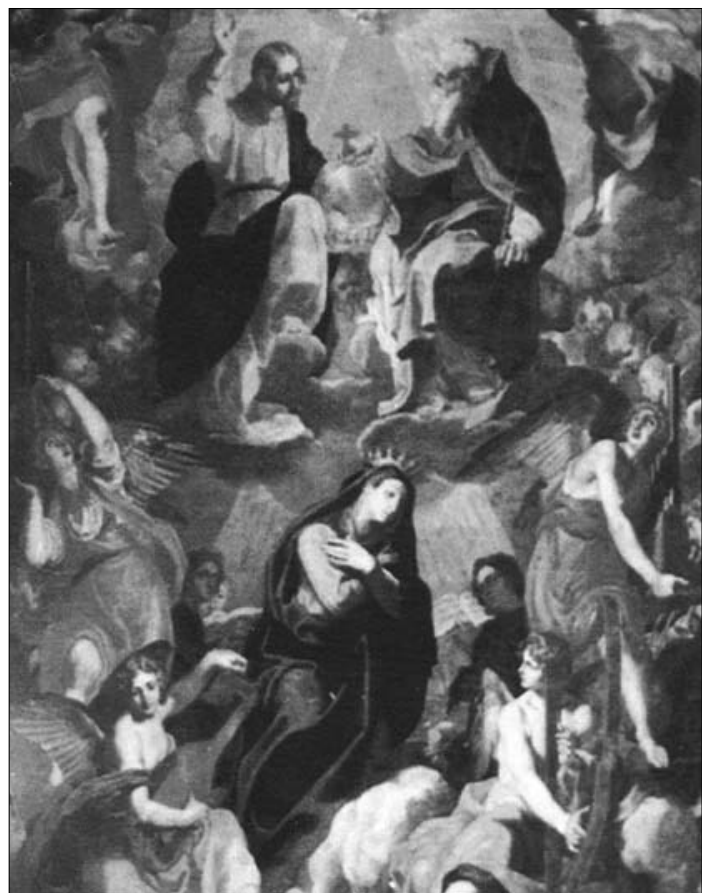

Fig. 1. Andrea Lilio, Asunción de la Virgen, Sevilla, Alcazar, Capilla del Palacio Gótico.

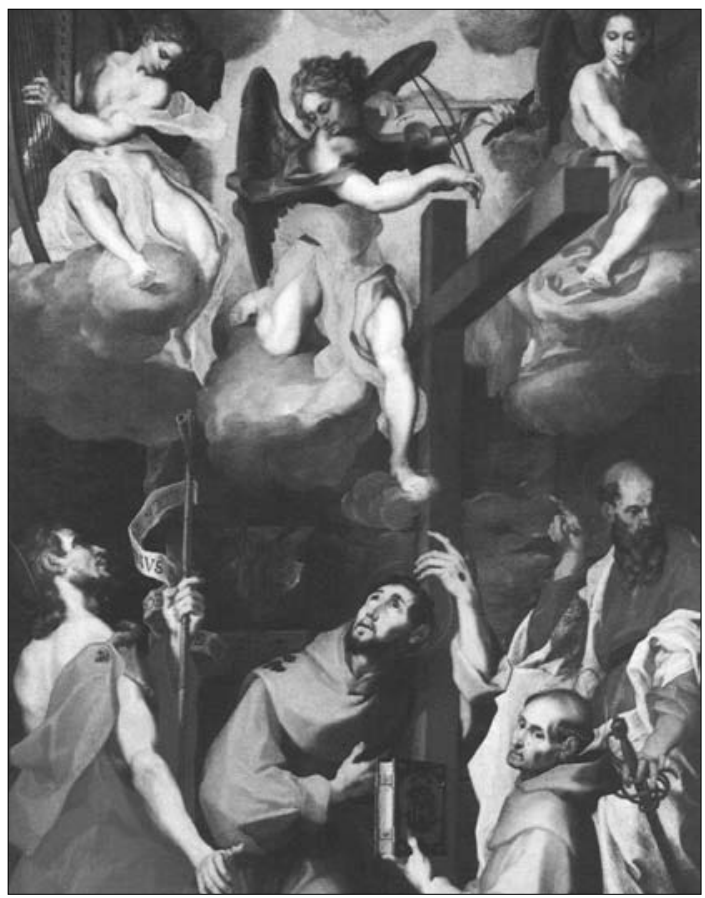

Fig. 3. Andrea Lilio, Cuatro santos con ángeles músicos, Ancona, Pinacoteca Comunale.

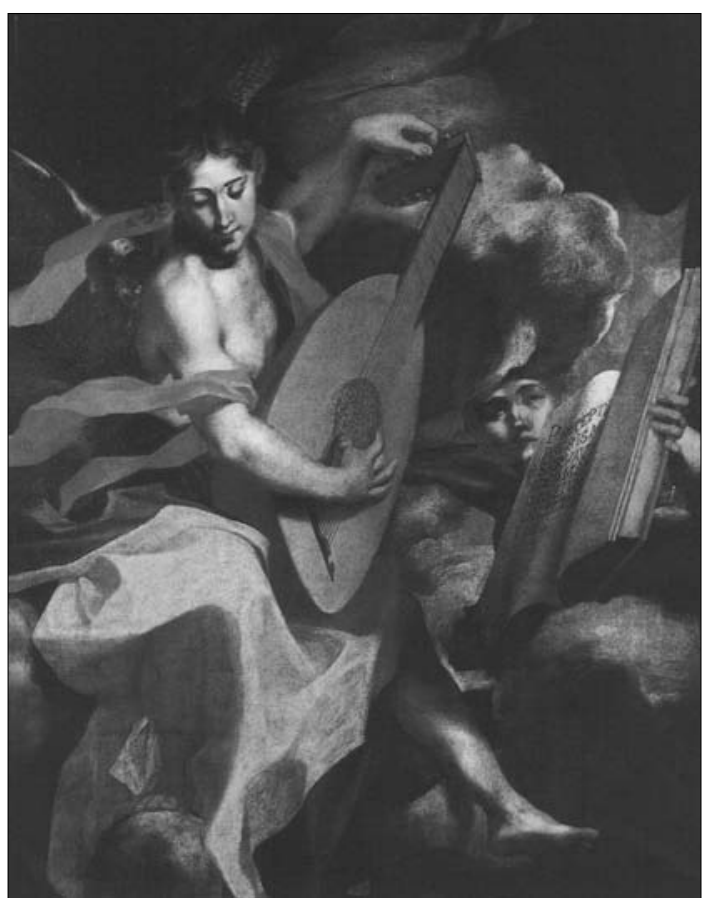

Fig. 2. Andrea Lilio, Ángeles músicos, Ancona, Pinacoteca Comunale.

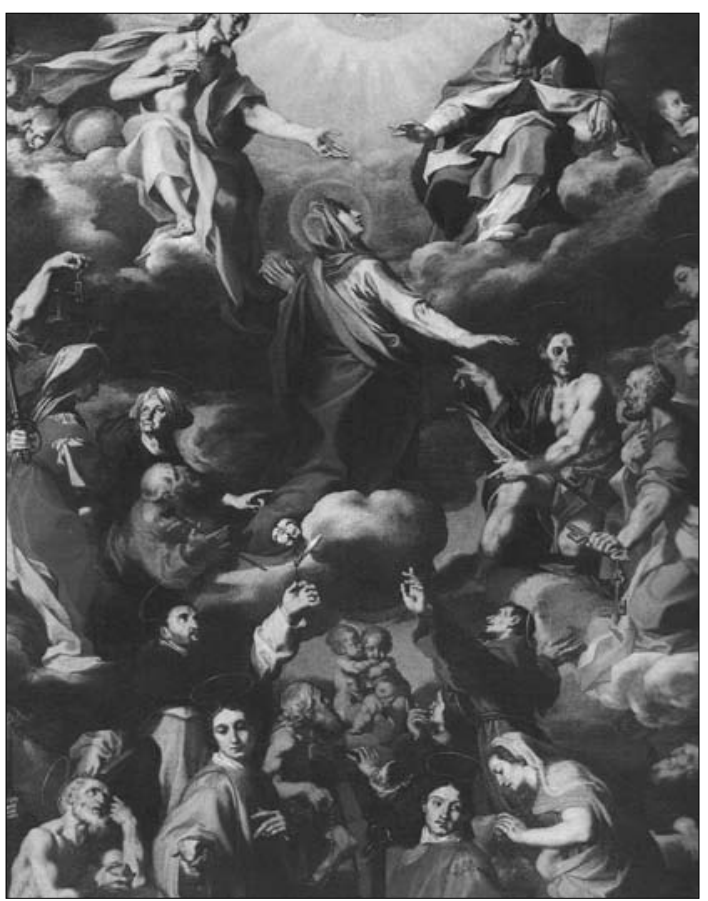

Fig. 4. Andrea Lilio, Asunción de la Virgen,

Fano, Duomo, Capilla Nolfi. 
Podemos por tanto establecer la hipótesis de que la gran tela de la Coronación de la Virgen llegó al Alcázar solo a principios del siglo XX, como por otra parte sucede con otros cuadros más célebres 6 . Las investigaciones deben entonces dirigirse hacia el territorio de la propia Sevilla, aunque toda posible búsqueda ha resultado bastante ardua por la compleja historia del patrimonio eclesiástico andaluz, objeto de varias y cruentas supresiones en el transcurso del siglo XIX ${ }^{7}$.

Entre los cuadros reunidos de iglesias y conventos de Sevilla en las salas del Alcázar después de la invasión napoleónica de la ciudad en 1810, para ser enviados al Museo Napoleón que se estaba constituyendo, futuro Louvre, o para ser utilizados como mobiliario en residencias y despachos del nuevo gobierno, figura "Un quadro de 4 varas de alto y 2 1/4 de ancho, la coronación de N. ra Señora", de autor desconocido, referencia que, sin embargo, ofrece pocos motivos para la identificación segura del cuadro 8 .

Una huella menos fiable en este sentido podría estar en cambio constituida por una noticia proporcionada por el propio Félix González de León, quien al describir el convento sevillano de la Concepción de San Miguel, identificado como pequeño y ruinoso, recuerda que en la iglesia "Al lado del Evangelio había dos malos retablos y al de la Epistola estaba el comulgatorio con un gran Lienzo que figuraba la Santissima Trinidad coronando a la Virgen, muy bien dibujado no tan bien pintado" 9 . El convento, unido a la parroquia de San Miguel hoy destruida y consignado a las monjas Concepcionistas (Terciarias franciscanas), fue suprimido en 1837; las hermanas se unieron a las del monasterio del Socorro y todos los objetos de culto fueron ofrecidos al "Comisionado de Amortización". La iglesia, a causa de los daños sufridos en los combates de 1834, fue desacralizada y destinada a cocheras reales, mientras que las salas conventuales se transformaron en almacenes y talleres 10 .

No se puede, por otra parte, excluir del todo que el cuadro provenga de los bienes de la corona conservados en Madrid o que pueda haber sido incluso una adquisición de Isabel II, como en el caso de San Francisco penitente de Luis Tristán, ya en la colección del Marqués de Salamanca, también éste hoy del patrimonio del Alcázar ${ }^{11}$.

En realidad, aunque no tengamos noticia alguna de los viajes de Lilio a España o a otro lugar - toda su carrera conocida se desarrolla entre Roma y Las Marcas -, la breve afirmación de Baglione (1642) que recuerda como "Dipinse anche altri quadri per alcuni luoghi fuori di questa mia Patria" deja suponer que obras del pintor de Ancona llegaran a España ya desde principio del siglo XVII.

6 Por ejemplo La imposición de la casulla a San Ildefonso de Velázquez, hoy en depósito en la misma capilla del Alcázar, en 1925 era custodiado en los aposentos privados del Palacio Arzobispal (cfr. Guichot Y SERRA, A., El Cicerón de Sevilla, 1925, I, p. 163) y luego fue donado por el arzobispo Bueno Monreal al Ayuntamiento de Sevilla, mientras que la Virgen del Buen Aire proveniente de la Casa de la Contratación fue recuperada desmembrada en la capilla del Alcázar y enviada a Madrid en 1925, para después volver al Alcázar (ídem, II, p. 36).

7 Un trabajo útil sobre el tema de las desamortizaciones sevillanas es el volumen de Fraga IrIBarne, M. L., Conventos femeninos desaparecidos. Arquitectura religiosa perdida durante el siglo XIX en Sevilla, Sevilla 1993.

8 GómEz IMAZ, M., Inventario de los cuadros sustraídos por el gobierno intruso en Sevilla el año de 1810, Sevilla 1896, p. 63 (en la Sala Baja del Alcázar), n 39 (entre los "autores desconoscidos"). El cuadro explicado mediría por lo tanto (estando el valor de la vara castellana a 83,59 centímetros) 3,34x1,88 metros, con una diferencia de medidas y de proporciones respecto al de Lilio, lo que induciría a descartarlo. Como sucede en otros países europeos, con la restauración de la monarquía nacional en España en 1814, parte de los cuadros sustraídos fueron restituídos y parte permaneció en Francia, en el Louvre y en otros lugares.

${ }^{9}$ GonZÁlez De LeÓN, F., op. cit., 1844, I, p. 229.

10 Fraga IriBarne, M. L., op. cit., pp. 91-97.

11 Véase la ficha en la reciente exposición a cargo de Pérez SÁncheZ, A. E., NAVARrete Prieto, B., De Herrera a Velázquez. El primer naturalismo en Sevilla, Sevilla-Bilbao, noviembre 2005-mayo 2006, [2005], ficha 47, p. 230.

Podría en cambio excluirse la hipótesis (según las informaciones de Rocío Ferin del Patrimonio Nacional, quien ha consultado los inventarios) de que el cuadro provenga, como los otros guardados hoy en el Alcázar, desde el palacio del Lomo del Grullo, la residencia real en Huelva, a las dependencias administrativas de los Reales Alcázares de Sevilla; el edificio progresivamente abandonado en el transcurso del siglo XVIII, fue posteriormente vendido a los duques de Montpensier en el siguiente siglo, y finalmente a particulares por el infante Don Alfonso en el siglo pasado (véase HaLCón, F., «El palacio del Lomo del Grullo», Archivo Español de Arte (A.E.A.), vol. 65, n 257, 1992, pp. 79-84). 
La idea de que la tela provenga del convento de San Miguel, donde podía encontrarse ab antiquo, me parece en definitiva totalmente plausible, incluso a la luz de las intensísimas relaciones que unían el mecenazgo y el coleccionismo de arte andaluz a Italia en tiempos de Felipe III y Felipe IV, de los que los casos del Duque de Alcalá, Fernando Enríquez Afán de Ribera, y del Conde Duque de Olivares, Gaspar de Guzmán, son solo los ejemplos más notables de una historia que está todavía en gran parte por trazar ${ }^{12}$. El cuadro de Lilio entonces, podría haber sido encargado directamente por un prelado vinculado al monasterio de las Concepcionistas, o haber sido dejado en herencia a estas por cualquier colección de arte sevillana, como sucede en otros numerosos casos similares. El estado de las indagaciones sobre las relaciones artísticas entre Italia y Sevilla no permite ir más allá en las conjeturas, a la espera de que ulteriores investigaciones aclaren una materia tan prometedora y todavía en muchos sentidos inexplorada.

PATRICIA TOSINI

\footnotetext{
12 Una importante panorámica sobre las relaciones entre coleccionistas andaluces e Italia a principios del siglo XVII está en el ensayo de SALORT Pons, S., Las relaciones artísticas entre Italia y Sevilla durante el primer tercio del siglo XVII, en Pérez SÁnchez, A., Navarrete Prieto, B., (a cargo de), op. cit., [2005], pp. 53-68. El autor examinando los principales casos de marchantes y coleccionistas en Sevilla y alrededores, unidos con el ambiente artístico italiano, subraya cómo, a diferencia del contexto madrileño, mejor conocido, para Andalucía la investigación está todavía por hacer.
} 